\title{
Carcinogenesis in chronic ulcerative colitis: Flow cytometry
}

\begin{abstract}
Flow cytometry is a conceptually straightforward quantitative method that can be used to determine the DNA content (and many other parameters) of individual cells. It has been used in chronic ulcerative colitis to evaluate the presence of aneuploidy as a marker of colon cancer risk. In this paper, the basic principles of flow cytometry are reviewed, the current status of published studies using flow cytometric analysis of DNA content in chronic ulcerative colitis outlined, conclusions from the research are proposed and future research needs identified. Overall, aneuploidy correlates with the current best markers of cancer risk in ulcerative colitis, ie, dysplasia and established cancer. Whether aneuploidy will be useful for the prospective identification of individuals at increased risk for colon cancer deserves rigorous evaluation. An important technical issue of potential variability in interpretation of near diploid peaks is emphasized. Can J Gastroenterol 1990;4(7):390-396
\end{abstract}

Key Words: Cancer, Flow cytometry, Surveillance, Ulcerative colitis

\section{La carcinogenèse dans la colite ulcéreuse chronique et la cytométrie de flux}

RESUME: La cytométrie de flux est une méthode quantitative simple pouvant servir à déterminer le contenu d'ADN (parmi de nombreux paramètres) des cellules individuelles. Elle permet de repérer l'aneuploïdie en tant que marqueur de risque de cancer du côlon dans la colite ulcéreuse chronique. Le présent article passe en revue les principes élémentaires de la cytométrie de flux, évalue le statut présent des études publiées dans le domaine, présente leurs conclusions et définit les futurs besoins de la recherche. En général, il y a corrélation entre l'aneuploïdie et les meilleurs indices de risque de cancer dans la colite ulcéreuse, c'est-à-dire la dysplasie et le cancer établi. Il est nécessaire de procéder à un examen rigoureux afin de déterminer si l'aneuploïdie permettra de déterminer qui présentent un risque élevé de cancer colique. L'auteur soulève une question technique importante: celle de la variabilité potentielle d'interprétation des pics proches de l'état diploïde.

Department of Medicine, Denver Veterans Administration Medical Center, University of Colorado Health Sciences Center; Department of Pathology, Rose Medical Center Denver, Colorado; and Department of Medicine, Cleveland Clinic Foundation, Cleveland, Ohio

Correspondence and reprints: Dr DJ Ahnen, University of Colorado Health Sciences Center, 4200 East 9 th Avenue, Denver, CO 80262, USA
$\mathrm{T}$ HE PURPOSE OF THIS ARTICLE IS to review the application of flow cytometry to the problem of carcinogenesis in chronic ulcerative colitis. In this review, methodology will be described including the capacity and limitations of flow cytometry; the data concerning flow cytometric analysis of DNA content in chronic ulcerative colitis will be reviewed; reasonable conclusions will be drawn from existing data; and finally, some research needs in this area will be identified.

\section{FLOW CYTOMETRY METHODOLOGY}

The basic principle of flow cytometry is the ability to measure the intensity of fluorescence in an in. dividual cell or nucleus. As illustrated in Figure 1, isolated cells or nuclei from the tissue of interest are prepared and labelled with a fluorescent label. The fluorescent label may be a fluorescence. tagged antibody directed against a cel. lular constituent or, in the case of DNA analysis, a fluorescent dye that binds quantitatively to DNA. The fluorescence-labelled single cells are dispersed into single droplets of saline. The dispersed cells are passed in single file through a laser beam which excites the fluorescence, and a fluorescence detec. tor quantitates the intensity of fluores. cence in each individual cell. An electric charge may be applied to cells 


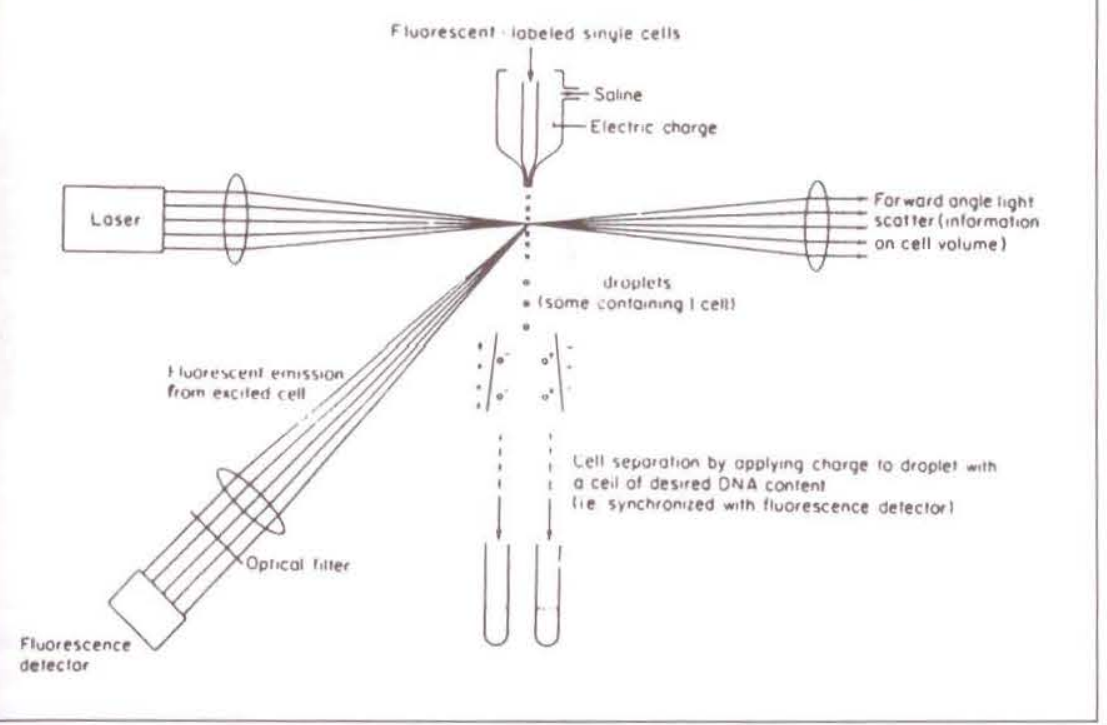

Figure 1) The principal features of a flow cytometer. Single cells are labelled with a fluorescent probe and directed in single file through a laser beam. Analysis of forward angle light scatter can be used to estimate cell volume. Fluorescence emission from the excited cell then provides a distribution of fluorescence intensity. Electric charge related to fluorescence intensity can also be applied to droplets containing single cells, allowing their separation in an electrostatic field. This process is known as 'fluorescence-activated cell sorting'

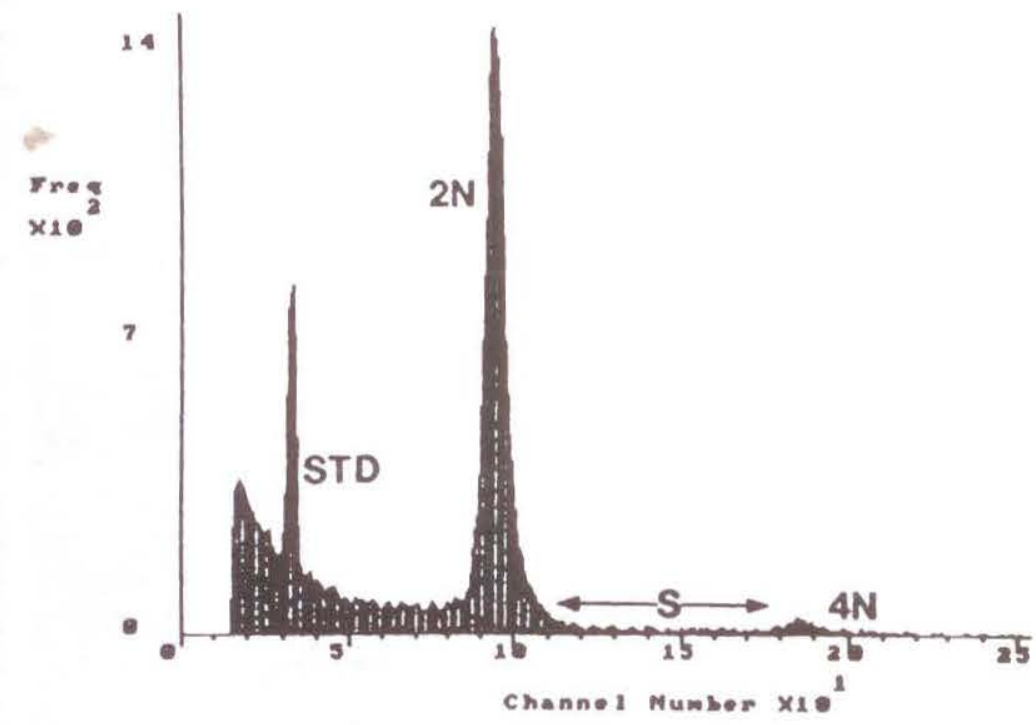

Figure 2) Flow cytometric analysis of DNA content. In this flow cytometric histogram the intensity of fluorescence is plotted on the horizontal axis and the number of cells on the vertical axis. An internal standard (STD) is run with each sample to allow quantitation of each of the fluorescent peaks. The major population of cells contains two copies of each chromosome (2N DNA). A smaller peak of cells with twice as much fluorescence contains four copies of each chromosome (4N DNA) and represents the cells in G2 and M phases of the cell cycle. The cells and assays of the cycle are represented by the cells between the $2 \mathrm{~N}$ and $4 \mathrm{~N}$ peaks

of differing fluorescence intensities so that they can be separated in an electrical field. This process is known as 'fluorescence-activated cell sorting'. In addition to fluorescence intensity, in- formation about cell volume can be obtained by the degree of forward angle light scatter of the excitation laser beam.

The flow cytometric data can then be displayed in a histogram (Figure 2), plotting the number of cells versus the intensity of fluorescence. Computer methods can be used to calculate the proportion of cells with any given intensity of fluorescence.

Almost all of the studies using flow cytometry in chronic ulcerative colitis have measured DNA content. For the use of flow cytometric analysis of DNA content, the isolated cells are incubated with fluorescent dyes such as propridium iodide, whose binding is proportional to the DNA content. The intensity of fluorescence is then proportional to the amount of DNA in each individual nucleus as shown in Figure 2. Generally an internal standard is run with each sample so that the amount of DNA in each peak can be quantitated. The major peak of cells in normal tissue contains two copies of each chromosome (2N DNA). These are the cells that are in G0 and G1 phases of the cell cycle. A small peak of cells can be seen having twice as much intensity as the $2 \mathrm{~N}$ peak. These cells contain four copies of each chromosome (4N DNA) and are the cells in the $\mathrm{G} 2$ and $M$ phase of the cell cycle. The cells synthesizing DNA (those in S phase) are seen as a plateau of cells between the $2 \mathrm{~N}$ and $4 \mathrm{~N}$ peaks.

Aneuploidy is defined as a population of cells containing an abnormal amount of DNA. An aneuploid cell population can be detected by flow cytometry as a peak of cells containing an abnormal amount of DNA that is discrete from the G0/G1 peak or the G2/M peak. Such an aneuploid population is indicated by the arrow in Figure 3. This peak is distinctly separate from the G0/G1 peak and has a DNA index which contains about 1.2 times the normal amount of DNA in diploid cells.

Flow cytometric analysis can be used for a large variety of techniques. Flow cytometry has the advantage of being a quantitative method in that it measures the amount of fluorescence in each individual cell. It also has the capacity to measure multiple fluorescent labels simultaneously using a dual beam flow cytometer. Thus, two or three quantitations can be made from the same cell using different fluorescent labels. For 
example, the amount of DNA and the intensity of growth factor receptor could be measured simultaneously to try to correlate growth factor expression with proliferation in individual cells. In addition, by measuring the forward angle light scatter, a measure of cell volume can be determined. Some flow cytometers have the capacity to sort populations of cells with varying amounts of fluorescence intensity, thus separating subsets of cells which can be studied further. Although the best results with flow cytometry are obtained when the tissue is obtained fresh and prepared immediately, archival blocks of paraffin-embedded tissue can be used for some studies, including DNA analysis. Thus, the presence of aneuploidy can be detected from such archival specimens.

There are also some important limitations to the use of flow cytometry. The technique must be standardized at each institution that develops flow cytometry as a clinical or research tool. This standardization includes not only the equipment but the selection of appropriate software for the interpretation of the flow cytometric results. In addition, for each separate application of flow cytometry to detect a different fluorescent label, the method must be standardized so that normal 'control' levels are determined within the population at, each individual institution.

For analysis of DNA content, two particular problems (debris and aggregation) deserve mention. During preparation of tissue, some nuclei may be fractured and produce debris that registers at the low fluorescence intensity region of the DNA histogram. This debris curve can extend into the regions of the $2 \mathrm{~N}$ and $\mathrm{S}$ phases of the cell cycle and can variably alter the quantitative results of these cell kinetic parameters. Some software calculates a debris curve and automatically subtracts it from the $2 \mathrm{~N}$ or $\mathrm{S}$ phase calculations whereas other software does not; therefore the percentage of cells in the $S$ phase, for example, could vary substantially depending upon the type of software used between two institutions. Aggregates of nuclei that are suspended

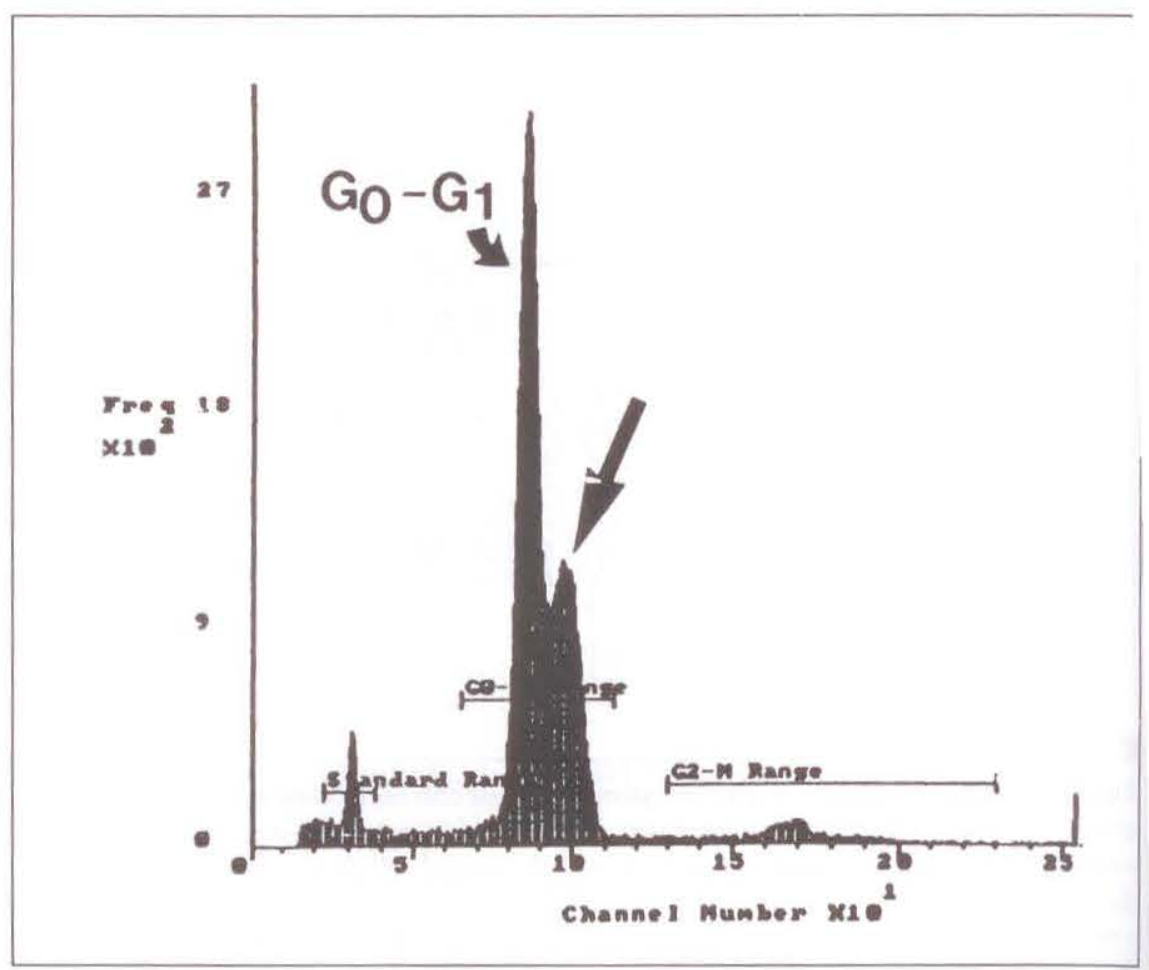

Figure 3) This DNA histogram shows a distinct peak of cells (indicated by the arrow) that is separate from the major GO/G1 peak. This population of cells contains approximately 1.2 times the amount of DNA in the GO/GI peak, would be classified as a near diploid aneuploid population

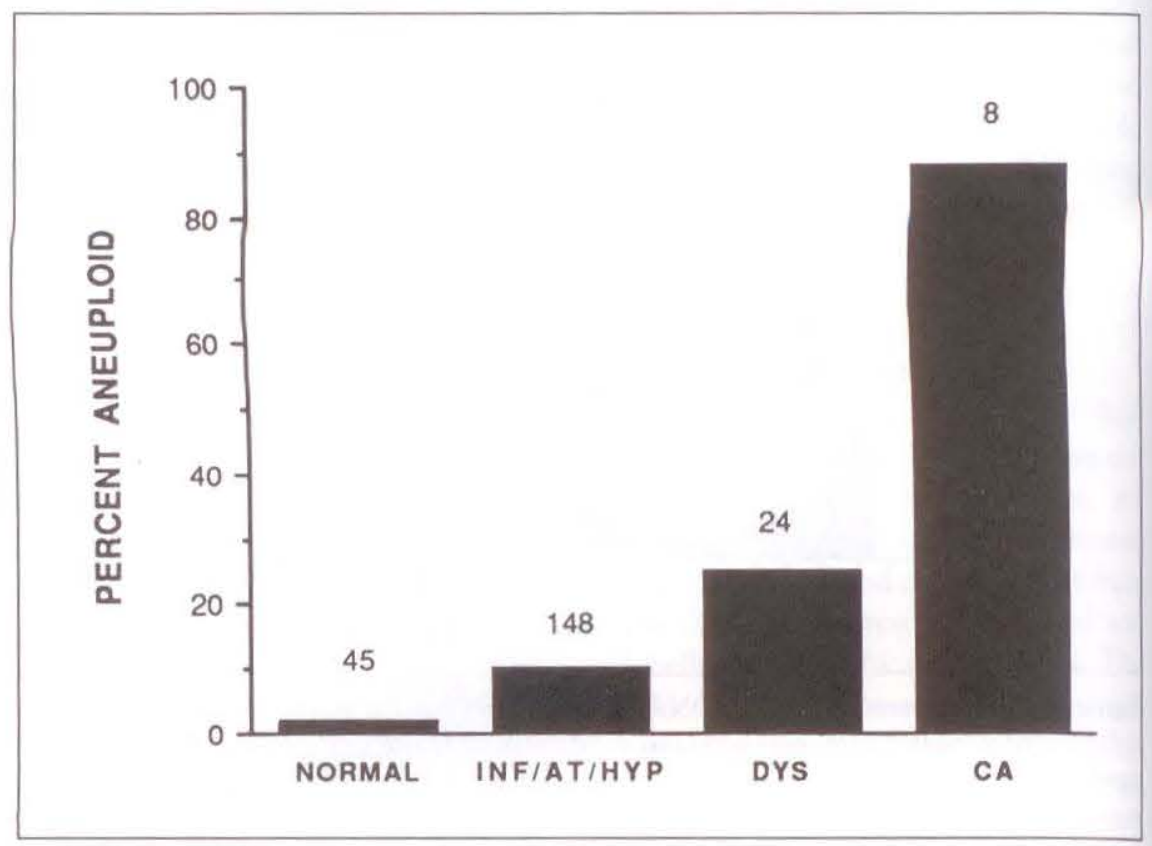

Figure 4) The frequency of aneuploidy in chronic ulcerative colitis. The percentage of biopsies that are aneuploid is plotted for groups of biopsies that are interpreted as normal; inflamed, atrophic or hyperplastic (INF/AT/HYP); dysplastic (DYS); or cancer (CA). The number above each column represents the number of biopsies analyzed in each group

in a single droplet of saline can be registered as containing two, three or more times the amount of DNA as a diploid peak. Doublets would be recorded as an increase in the $4 \mathrm{~N}$ peak and could potentially be misinterpreted as a large tetraploid population. It is important to recognize this possibility 


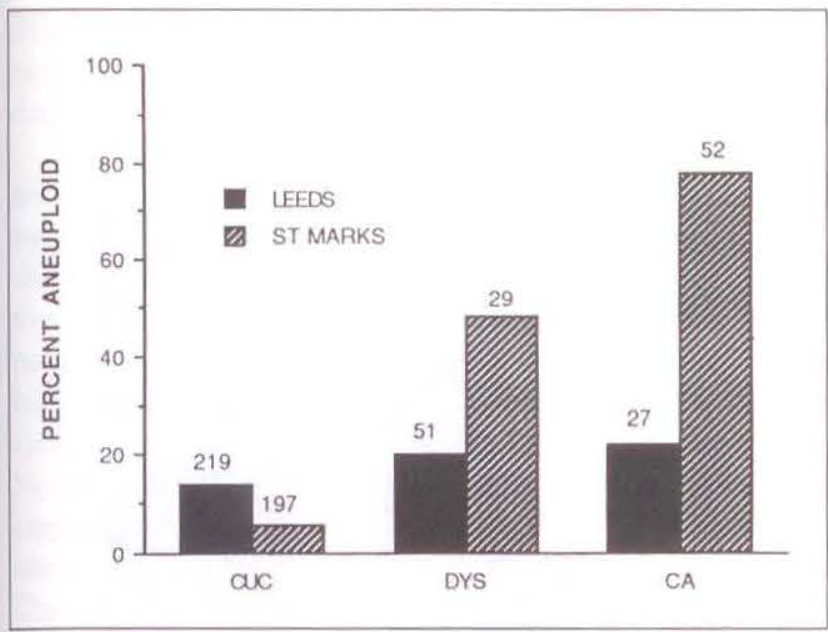

Figure 5) The frequency of aneuploidy in chronic ulcerative colitis biopsies from two studies from the United Kingdom. The data from the Leeds' group (3) is shown as solid bars and that from the St Mark's group (4) as cross-hatched bars. The percentage of biopsies that are aneuploid is plotted for each of three groups of biopsies interpreted as showing only ulcerative colitis without dysplasia (CUC), dysplasia (DYS) and cancer (CA). The numbers at the top of each column are the numbers of biopsies analyzed in each group

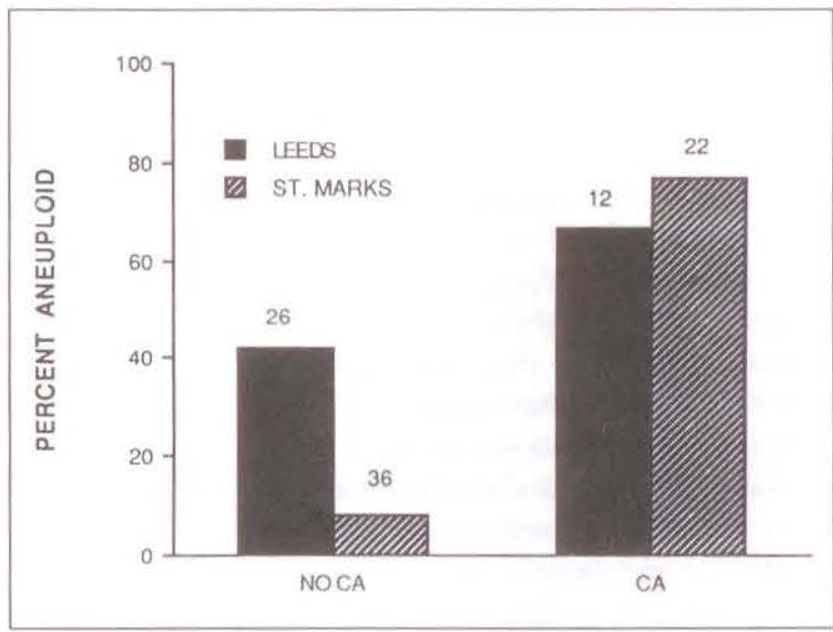

Figure 6) The frequency of aneuploidy in any biopsy from patients with and without carcinoma from two studies from the United Kingdom. The study from Leeds (3) is shown as closed bars, while those from St Marks (4) are shown as cross-hatched bars. The percentage aneuploidy in any biopsy is plotted for two groups of patients, those having no evidence of cancer in any biopsy (NO CA) and those having cancer in one or more biopsies (CA). The number at the top of each bar represents the number of patients in each group and to expand the vertical scale, if necessary, to look for triplets in any histogram showing a large tetraploid population. If aggregation is the problem, one will see a smaller peak of cells containing $6 \mathrm{~N}$ DNA (triplets). In this case, the sample should be resuspended and re-analyzed to validate the results. As with any other technique, flow cytometry has to be set up carefully and monitored in order to be reproducible and of clinical value.

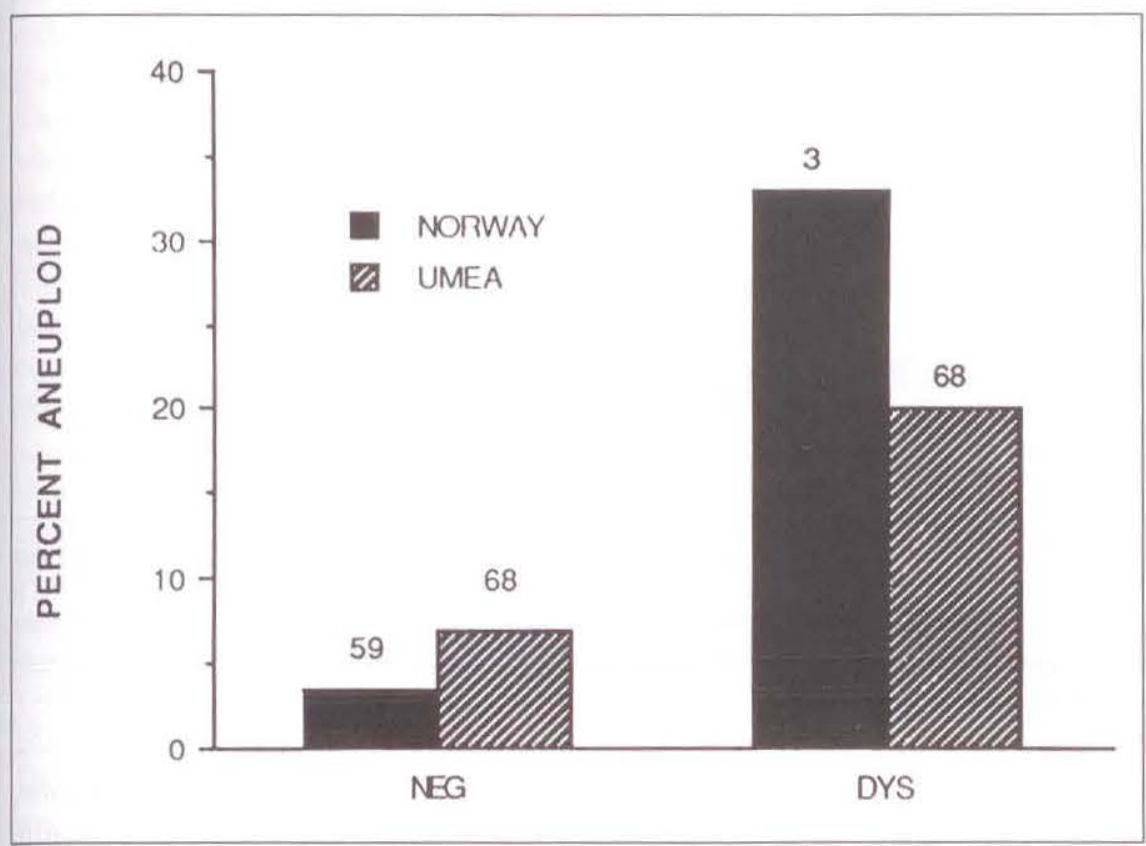

Figure 7) The frequency of aneuploidy in biopsies from patients with chronic ulcerative colitis from two studies is presented. The study from Bergen, Norway is shown as closed bars and the study from Umea, Sweden is shown as cross-hatched bars. The percentage of biopsies that are aneuploid is plotted for those graded as negative for dysplasia (NEG) and those interpreted as dysplastic (DYS). The number of biopsies in each group is shown above the bars

\section{FLOW CYTOMETRIC ANALYSIS OF DNA CONTENT IN CHRONIC ULCERATIVE COLITIS}

The initial work that stimulated interest in flow cytometric analysis of DNA content in chronic ulcerative colitis came from Hammarberg et al $(1,2)$. These authors analyzed biopsies from patients with ulcerative colitis and found a high frequency $(90 \%)$ of aneuploidy in colon cancers in the setting of ulcerative colitis and a low (less than $5 \%$ ) frequency of aneuploidy in normal tissue. Intermediate levels of aneuploidy were identified in dysplastic mucosa and inflamed atrophic or hyperplastic mucosa in chronic ulcerative colitis (Figure 4). Two subsequent studies from the United Kingdom, however, came to divergent conclusions $(3,4)$. The results of analysis of individual biopsies in these two studies is plotted in Figure 5. The study from Leeds showed no significant difference in the frequency of aneuploidy from biopsies showing ulcerative colitis only, dysplasia, or cancer, with a frequency between 18 and $24 \%$ (3). In contrast, a gradient of increase in frequency of aneuploidy in these three biopsy classifications was seen by the St Mark's group (4). These observations were par- 
ticularly disturbing because the studies were done in a very similar manner using archival fixed and paraffin-embedded tissues, and using similar flow cytometric techniques. Furthermore, it was initially difficult to envision a systematic difference in preparation or interpretation that might account for the differences because the Leeds group found both a higher frequency of aneuploidy in the biopsies showing no dysplasia or cancer, and a substantially lower frequency of aneuploidy in the dysplastic and cancer biopsies. If there was a systematic difference in the detection or interpretation of aneuploidy, one would expect to show consistent differences in the same direction in all of the groups rather than this disparate result. Subsequently, however, the Leeds group reported that their frequency in cancer had increased to about $46 \%$ so that the disparity seemed to be more in the biopsies graded negative for dysplasia or cancer. This is also the case if one analyzes the data with respect to the number of patients rather than the individual biopsies (Figure 6). The percentage of patients with cancer that had aneuploidy was similar between the Leeds and St Marks studies; however the Leeds group found that over $40 \%$ of patients with no evidence of cancer had aneuploidy, whereas it was less than $10 \%$ in the study from St. Marks. Thus, it seems that the major difference between the two studies is the finding of a higher frequency of aneuploidy in the non-neoplastic biopsies in patients by the group at Leeds compared to $\mathrm{St}$ Marks. It is possible that a difference in the criteria used for the classification of aneuploidy between the two studies could account for such differing results.

There have been several other studies that have presented data related to the frequency of aneuploidy in chronic ulcerative colitis. In Figure 7, the results of two studies reporting the correlation between aneuploidy and histology in individual biopsies are shown. In both the study from Bergen, Norway (5) and from Umea, Sweden (6) a substantially higher frequency of aneuploidy was seen in biopsies showing dysplasia than in those negative for

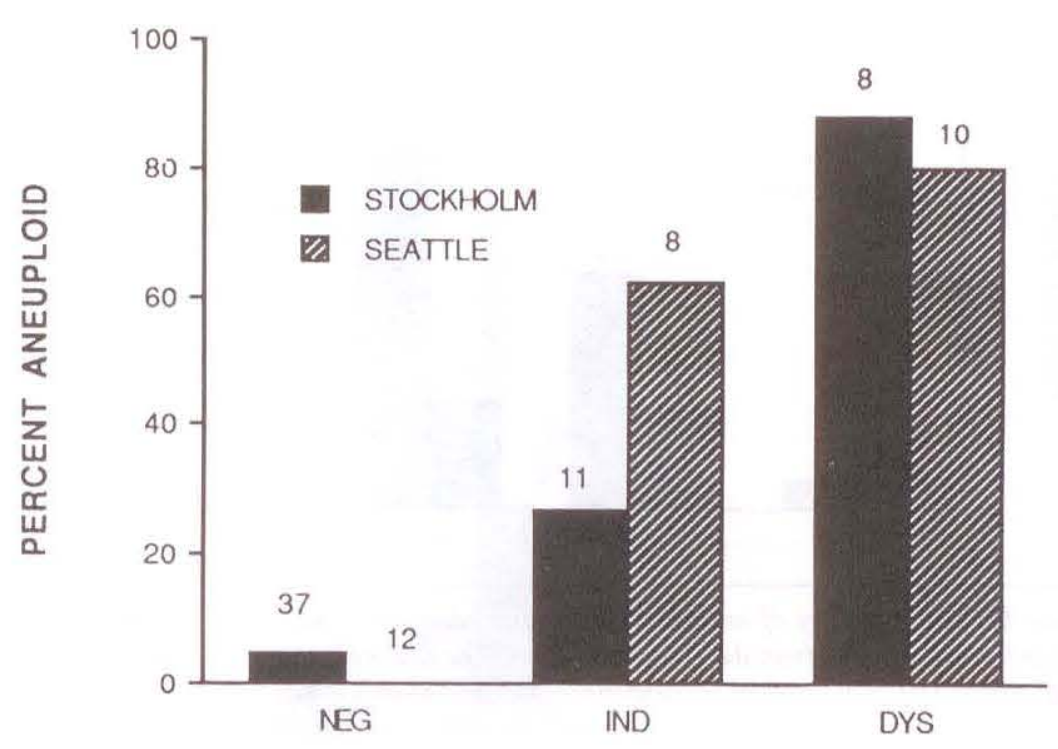

Figure 8) The frequency of aneuploidy in patients with chronic ulcerative colitis from two studies are presented. A study from Stockholm, Sweden (7) is shown as closed bars and a study from Seattle, Washington (8) as cross-hatched bars. The frequency of aneuploidy in three patient groups is plotted: patients whose biopsies were all graded as negative for dysplasia (NEG), those with one or more biopsies graded as indefinite for dysplasia (IND), and patients with one or more biopsies graded as dysplastic (DYS). The number above each bar represents the number of patients in each group

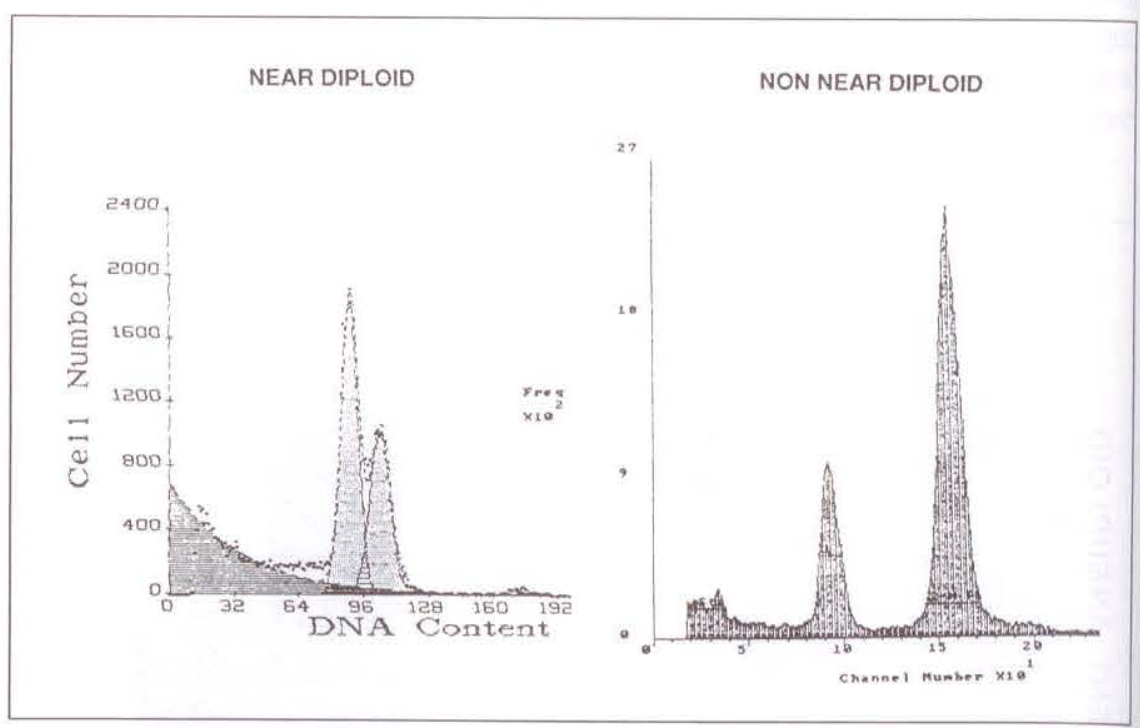

Figure 9) Patterns of 'aneuploid' peaks. These two histograms demonstrate different patterns that could be interpreted as aneuploidy. On the left, a population of cells with a DNA index of 1.2 is plotted. There is a discrete peak of cells with fluorescence intensity approximately 1.2 times the major $\mathrm{G} 0 / \mathrm{G} 1$ peak. On the right, a large aneuploid population is seen with a DNA content approximately 1.7 times the normal diploid peak

dysplasia. It is of some interest that the absolute frequency in the negative group differed by almost two-fold between the two studies. Two additional groups have reported the results of the relationship between aneuploidy and histology analyzed by patient groups
(Figure 8). The study from Stockholm, Sweden (7) and the study from Seattle (8) both show a gradient of increasing frequency of aneuploidy in patients who only have biopsies that are negative for dysplasia, those that have one or more biopsies graded as indefinite for 
dysplasia, and those that have one or more biopsies graded as definite dysplasia.

The bulk of the evidence in the literature regarding the relationship between aneuploidy and dysplasia in chronic ulcerative colitis does suggest that biopsies that are dysplastic and patients who show dysplasia have a higher frequency of aneuploidy than those with no evidence of dysplasia. Nonetheless, there is significant discrepancy concerning the frequency of aneuploidy in nondysplastic mucosa. The authors have had the opportunity to perform a large number of flow cytometric studies in patients with chronic ulcerative colitis. Patients evaluated in these studies include patients entered from the Denver Dysplasia in Ulcerative Colitis study as well as patients enrolled in the Cleveland Clinic Chronic Ulcerative Colitis registry.

In the authors' studies, colonic biopsies were obtained from three groups. Controls have come to colonoscopy for a variety of reasons, but were found to have no colonic neoplasia and no personal history of chronic ulcerative colitis, adenomas or colon cancer. The other two groups are patients with chronic ulcerative colitis who are either enrolled in a surveillance program or who have come to colectomy. The surveillance group are patients who have longstanding (greater than eight years), extensive (proximal to the splenic flexure) ulcerative colitis. These patients are enrolled in surveillance studies in which yearly colonoscopy is advised and multiple biopsies obtained throughout the colon. The routine procedure in these patients is to obtain two adjacent biopsies every 10 $\mathrm{cm}$ throughout the colon as well as additional biopsies of any suspicious lesion. One of the biopsies at each site is fixed and prepared for histology and evaluation of dysplasia; the second is prepared for flow cytometric analysis of DNA content. Thus far over 850 biopsies from 80 patients in the surveillance group have been evaluated. The colectomy group includes patients with longstanding ulcerative colitis who have come to colectomy for intractable disease, dysplasia or cancer. In this group, two side-by-side four-quadrant biopsies are taken every centimetre through the entire resected specimen. As in the surveillance group, one biopsy at each site is prepared for flow cytometry, the other for histology. Thus far, over 1300 biopsies from 12 patients in this group have been evaluated.

The initial criteria used to define aneuploidy was the presence of a discrete peak of cells distinct from the G0/G1 and G2/M peaks that contained at least $5 \%$ of the total cell population. Using these criteria alone, a high frequency of aneuploidy was found (about $15 \%$ of the biopsies). Forty per cent of patients had one or more biopsies that were aneuploid. A large percentage of these putative aneuploid peaks are near diploid peaks, that is, they are discrete peaks, but they have DNA contents less than 1.2 times the diploid peak (Figure 9).

In the surveillance group, over $80 \%$ of the aneuploid biopsies not showing dysplasia fell into the 'near diploid' group. The frequency of aneuploidy in the nondysplastic tissue is thus influenced greatly by whether one interprets the near diploid peaks as aneuploidy. Interobserver variation in the interpretation of near diploid peaks has been noted in other tissues (9). This difference may well account for much of the variability in the literature cited above. As in the other studies in literature, the present authors achieved a highly significant increase in the frequency of aneuploidy both in dysplastic biopsies and in patients who have dysplasia or cancer.

Two other encouraging aspects of these studies should be mentioned. First, patients who come to colectomy because of dysplasia or cancer are very frequently found to have markedly abnormal flow cytometric analysis with multiple areas of aneuploid populations of different DNA index. For example, one of the patients studied came to colectomy because of a cancer found at the hepatic flexure. Multiple areas of dysplasia were seen in the splenic flexure as well as in the transverse colon. The majority of these dysplastic biopsies were aneuploid in addition, there were many nondysplastic biopsies that were also aneuploid. Overall, nine separate aneuploid populations were identified in this colonic mucosa. The presence of multiple aneuploid populations in patients with dysplasia and cancer has also been seen by several other groups who have examined this question. These observations suggest that in the late stages of carcinogenesis in chronic ulcerative colitis, aneuploidy is a prominent feature of the colonic mucosa. Secondly, the authors have been encouraged by the frequent finding that not only dysplastic biopsies show aneuploidy, but the same aneuploid population can often be seen in nondysplastic mucosa adjacent to dysplasia. For example, one patient had two biopsies at the hepatic flexure which showed dysplasia and were aneuploid with a DNA index of about 1.7. In addition, however, four adjacent nondysplastic biopsies showed the same aneuploid population. This type of observation suggests the possibility that aneuploidy may appear as a type of field effect and, if this is so, could decrease the problem with sampling error when looking for dysplasia. These observations encourage continued evaluation of aneuploidy as a promising marker of cancer risk in chronic ulcerative colitis.

\section{CONCLUSIONS}

The current data suggest that aneuploidy occurs commonly in chronic ulcerative colitis and that it correlates with the established markers of cancer risk in this setting. Aneuploidy is more common in patients with dysplasia and cancer, but not all patients with neoplasia have detectable aneuploidy. Aneuploidy is also more common in dysplastic and cancerous biopsies than in nondysplastic mucosa, but not all neoplastic biopsies are aneuploid. Aneuploidy also clearly occurs in non-neoplastic mucosa. The clinical meaning of the presence of aneuploidy in nondysplastic mucosa is not yet known and the authors do not currently recommend altering clinical decision making on the basis of a single aneuploid population in a patient with ulcerative colitis. 
Nonetheless, aneuploidy is the most promising of the new markers for the detection of cancer risk in ulcerative colitis and deserves further evaluation.

The data also suggest that aneuploid populations can appear over wide expanses of the colonic mucosa. This kind of observation can be viewed as an example of clonal expansion in this mucosa. It is possible that the ulceration and denudation of the mucosa that occurs in ulcerative colitis allows the opportunity for a clone of cells that has growth advantage to repopulate large areas of the colonic mucosa. In the absence of such denudation, perhaps this abnormal clone would be limited to a single crypt and the cells would be sloughed off into the lumen as they normally are. In the context of multistage carcinogenesis, one can view the appearance of aneuploidy in multiple areas of the colon as an example of such clonal expansion. If this aneuploid population were to represent one of the genetic events responsible for a step in carcinogenesis, its expansion over a wider area of the colon would mean that there would be a larger number of cells now available for subsequent carcinogenic steps, making cancer more probable. The most commonly held view is that aneuploidy does not itself represent a specific genetic event necessary for carcinogenesis, but is a

ACKNOWLEDGEMENTS: The authors acknowledge the many gastroenterologists and surgeons in the Denver and Cleveland areas who have contributed case material for these studies. Without them, this work would not be possible.

\section{REFERENCES}

1. Hammarberg C, Rubio C, Slezak P. Tribukait B, Ohman U. Flowwcytometric DNA analysis as a means for early detection of malignancy in patients with chronic ulcerative colitis. Gut 1984;25:905-8.

2. Hammarberg C, Slezak P, Tribukait B. Early detection of malignancy in ulcerative colitis. A flow-cytometric marker of the genomic instability that characterizes premalignant mucosa.

Analysis of the present data suggests that variable interpretation of the near diploid peaks may account for some of the differences reported in the literature about the relationship between aneuploidy, dysplasia and cancer in chronic ulcerative colitis. Inclusion of the near diploid populations in the authors' data markedly increases the overall frequency of aneuploidy, particularly in the nondysplastic group.

\section{RESEARCH NEEDS}

From this review of the literature and the present data, it appears that there are several important research needs that should be addressed. From a technical standpoint, it is important to attempt to define more precisely the significance of the near diploid populations. Such populations could be due to near diploid aneuploidy or they could represent something else, such as differential dye binding. For example, a population of cells might more avidly bind the fluorescent dye and thus have a marginally higher intensity of fluorescence, appearing as a near diploid peak. In addition, methods of preparation of cells could conceivably produce such near diploid peaks.

To compare flow cytometric analyses from different centres, it will

DNA study. Cancer 1984;53:291-5.

3. Fozard JB], Quirke P, Dixon MF, Giles GR, Bird CC. DNA aneuploidy in ulcerative colitis. Gut 1986;27:1414-8.

4. Melville DM, Jass JR, Shepherd NA, et al. Dysplasia and deoxyribonucleic acid aneuploidy in the assessment of precancerous changes in chronic ulcerative colitis. Observer variation and correlations. Gastroenterology 1988;95:668-75.

5. Borkje B, Hostmark J, Skagen DW, Schrumpf E, Laerum OD. Floww cytometry of biopsy specimens from ulcerative colitis, colorectal ademomas, and carcinomas. Scand J Gastroenterol 1987;22:1231-7.

6. Rutegard J, Ahsgren L, Stenling R, Roos G. DNA content in ulcerative be important to standardize both the methodology (sample preparation and staining) and the software used to interpret the flow histograms. This is particularly important when trying to compare the meaning of quantitative results between different flow cytometry centres.

In the clinical area, the most press. ing need is for follow-up studies of patients with aneuploidy with or with. out dysplasia to determine whether aneuploidy is predictive of current and/or subsequent colon cancer risk. This can be done in the setting of ongoing prospective trials, but in addition, a multicentre case control study using archival specimens seems feasible and could potentially answer this question more quickly.

\section{SUMMARY}

The emerging literature using flow cytometry in the setting of ulcerative colitis has thus far primarily focused on measurement of DNA content and aneuploidy. These studies have shown a significant relationship between aneuploidy and the current best markers of cancer risk in this popula. tion. Aneuploidy certainly deserves rigorous testing to determine if its presence will be of clinical value in assessing cancer risk in patients with chronic ulcerative colitis.

colitis. Flow cytometry analysis in a patient series from a defined area. Dis Colon Rectum 1989;32:1055-9.

7. Löfberg R, Tribukait B, Öst A, Broström O, Reichard H. Flow cytometric DNA analysis in longstand. ing ulcerative colitis: A method of prediction of dysplasia and carcinoma development? Gut 1987;28:1100-6.

8. Levine DS, Reid BJ, Haggitt CE, Rubin PJ, Dean PJ, Rabinovitch PS. Frequency and distribution of aneuploid cell populations in chronic ulcerative colitis. Gastroenterology 1988;94:A260.

9. Joensuu H, Kallioniemi O-P. Different opinions on classification of DNA histograms produced from paraffin-embedded tissue. Cytometry 1989;10:711-7. 


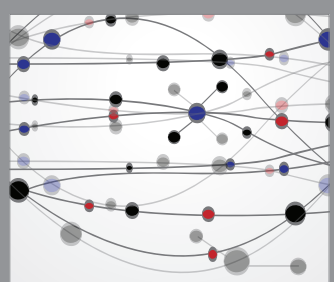

The Scientific World Journal
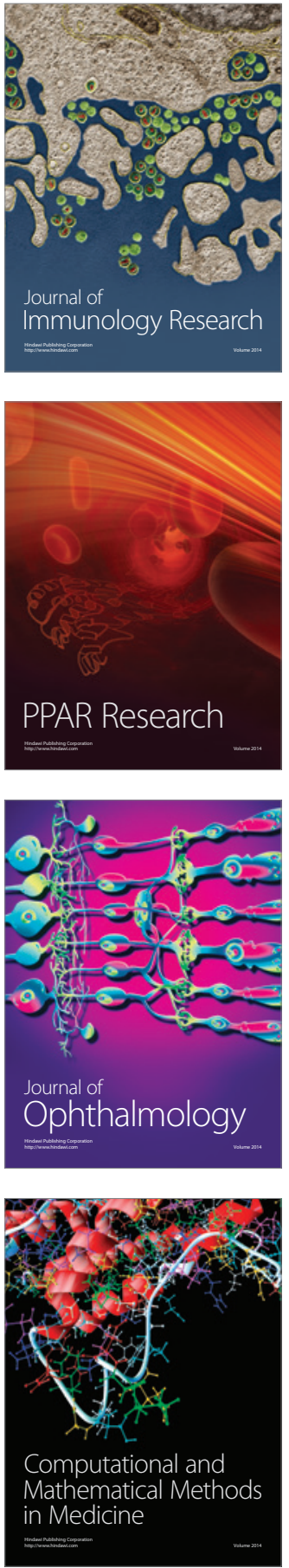

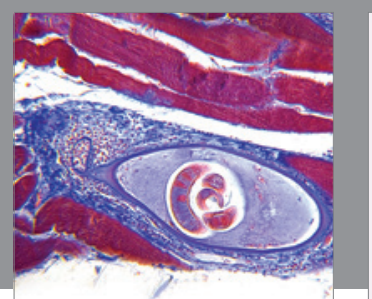

Gastroenterology Research and Practice

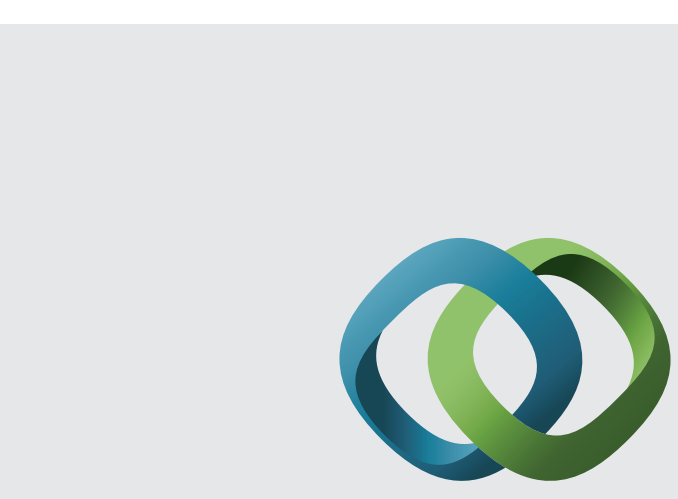

\section{Hindawi}

Submit your manuscripts at

http://www.hindawi.com
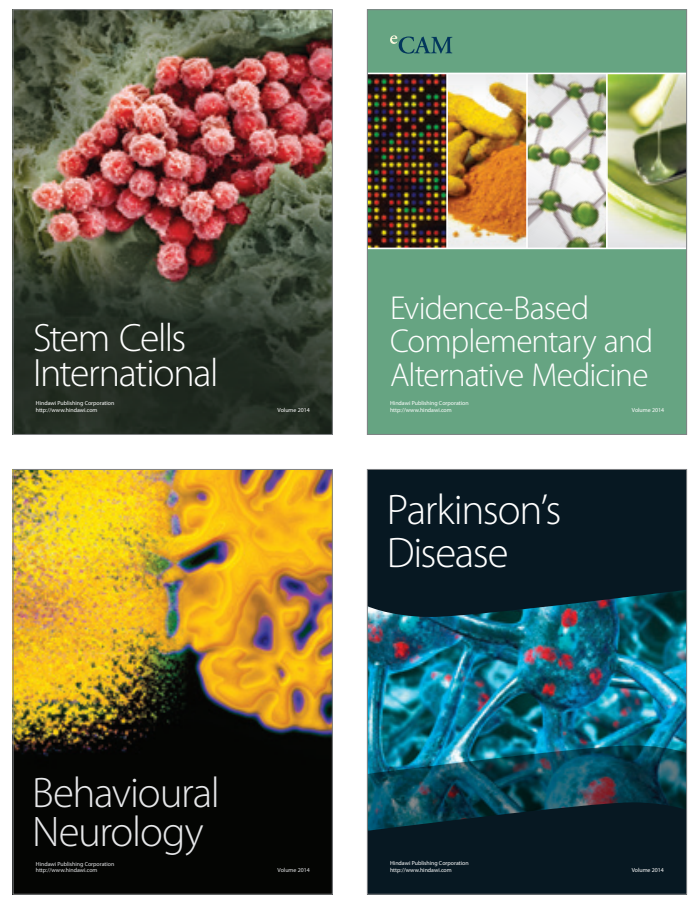
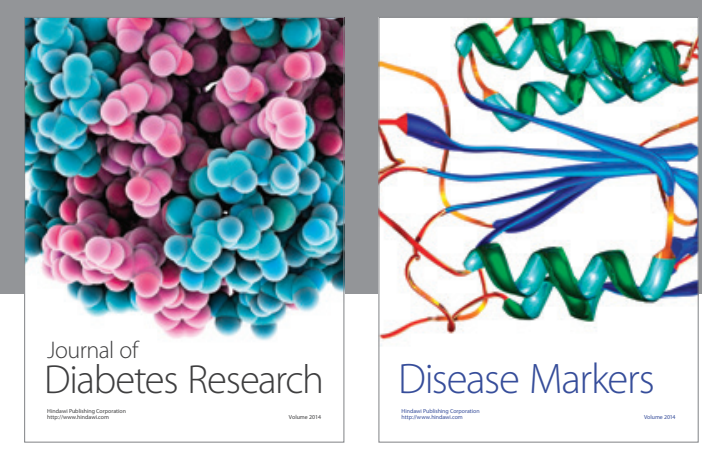

Disease Markers
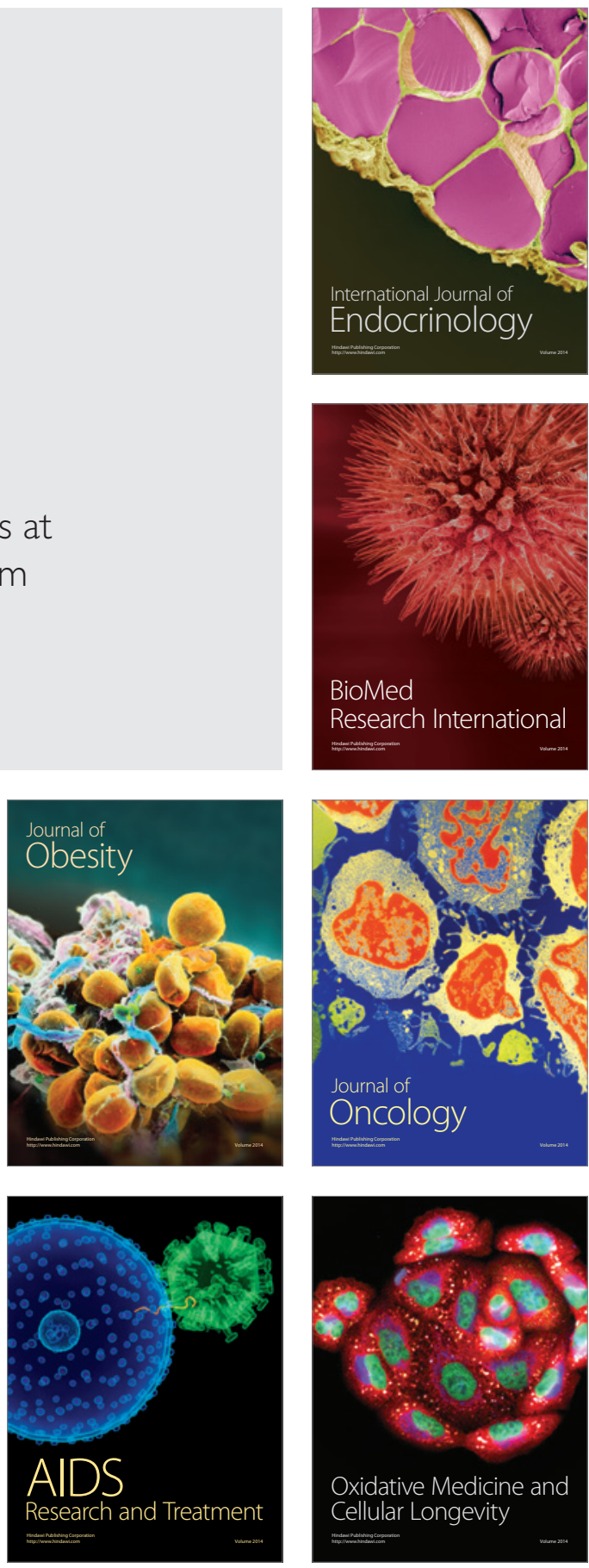